\title{
A flow approach to upward drawings of toroidal maps
}

\author{
Bojan Mohar* \\ Department of Mathematics \\ University of Ljubljana, Slovenia \\ and \\ Pierre Rosenstiehl ${ }^{\star \star}$ \\ Centre d'Analyse et de Mathématique Sociales \\ EHESS, Paris, France
}

\begin{abstract}
The model of the torus as a parallelogram in the plane with opposite sides identified enables us to speak of upward drawings of graphs in the torus. It is shown that upward embeddings of a toroidal map can be obtained in a very natural way by means of flow techniques.
\end{abstract}

\section{Definitions}

Given a graph $G$, let $V(G)$ be the set of vertices of $G, E(G)$ the set of edges of $G$. For $A, B$ subsets of $V(G)$, let $E(A)$ be the set of edges of $G$ with both ends in $A$, and let $E(A, B)$ be the set of edges of $G$ wilh one end in $A$, the other in $B$.

A map $M$ on a surface $S$ is a connected graph $G$ together with a 2-cell embedding of $G$ in $S$. Two maps are equivalent if there is a homeomorphism of $S$ mapping the graph of the first map onto the graph of the second. It is wellknown that the equivalence classes of maps on orientable surfaces correspond naturally to rotation systems on the underlying graphs [5], where a rotation system on a graph $G$ is a set of cyclic rotations of edges emanating from each vertex of $G$, the local rotations corresponding to the cyclic order of these edges on the surface. The rotation system around the faces of $M$ defines the dual map $M^{*}$. A map $M$ and its dual $M^{*}$ can be simultaneously drawn in $S$ such that each vertex of $M^{*}$ corresponds to an interior point of the corresponding face of $M$ (and vice versa), and such that precisely dual pairs of edges cross each other.

From now on we shall assume that the surface $S$ is the torus. An angle of a map $M$ (respectively, $M^{*}$ ) on $S$ is a pair of consecutive arcs at a vertex $v$ of $M$ (respectively, a face $f$ of $M$ ). The angle map of $M$ is a map $\mathcal{A}$ on $S$ whose vertices are the vertices plus the faces of $M$, and whose edges are the angles of $M$, each angle being incident with the corresponding vertex and face of $M$ [9]. The set

* Supported in part by the Ministry of Science and Technology of Slovenia, Research Project P1-0210-101-94.

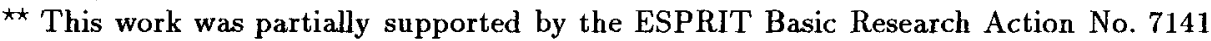
(ALCOM II). 
of angles incident with a vertex $v$ of $M$ has a local rotation determined by $M$, and the set of angles incident with a face of $M$ inherits the local rotation from $M^{*}$. Note that the angle map $\mathcal{A}$ is bipartite and each face of $\mathcal{A}$ is a quadrangle whose diagonals are a pair of dual edges of $M$ and $M^{*}$. An example is shown in Figure 1(b) where the dotted lines represent the edges of $M$ and they are not part of $\mathcal{A}$. The dual map of $\mathcal{A}$ is the medial map of $M$.

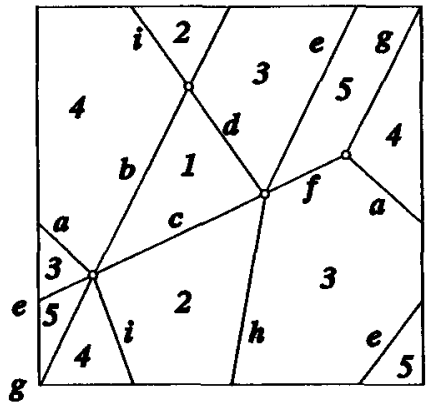

(a)

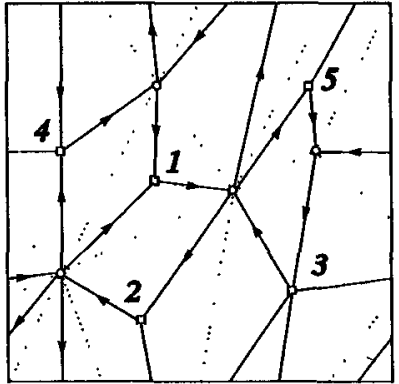

(b)

Fig. 1. A toroidal map and its angle map

A toroidal map $M$ is essentially 2-connected if for every subset $Y$ of the vertices of the angle map $\mathcal{A}$ of $M$ we have

$$
2|Y|-|E(Y)| \geq 0 .
$$

This property is equivalent to the requirement that the universal cover of $M$ is 2-connected [6]. Several other characterizations of this property are presented in [6].

For a given orientation of the edges of a map $M$, an angle at a vertex $v$ is lateral if one of its arcs is incoming and the other is outgoing at $v$. Otherwise, if both arcs of an angle are incoming or both are outgoing at $v$, then the angle is extremal. Having an orientation of edges of a map $M$, we get an orientation of the angle map $\mathcal{A}$ as follows. Given an angle $\alpha$ incident with a vertex $v$ and a face $f$ of $M$, the angle $\alpha$ as an edge of $\mathcal{A}$ is oriented from $f$ to $v$ if $\alpha$ is lateral, and from $v$ to $f$ if $\alpha$ is extremal. We say that an orientation of edges of $M$ has the upward property at $v$ (resp. at $f$ ) if there are exactly two lateral angles at $v$ (resp. two extremal angles at $f$ ). Equivalently, in the oriented angle map $\mathcal{A}$, the indegree of every vertex (either $v$ or $f$ ) is equal to two (see Figure 1(b)). Cf. also [7]. An orientation of $M$, which has the upward property at every vertex and every face, is called an upward orientation. By the above remark, an upward orientation induces an indegree two orientation of the angle map $\mathcal{A}$. It is an easy 
corollary of Euler's formula that only maps on the torus (and on the Klein bottle if we allow nonorientable surfaces) may accept an upward orientation. The aim of this contribution is the construction of upward orientations of a non-oriented toroidal map by means of a flow model.

Upward orientations are closely related to upward drawings. In order to define an upward drawing of a graph $G$ on the torus, we first need a definition of a monotone arc. Consider, without loss of generality, the flat torus that is obtained from a parallelogram $Q$ in the plane whose opposite sides are pairwise identified (see Figure 1). The lines parallel to the sides of $Q$ oriented according to the usual axes determine horizontal and vertical circuits on the torus. At each point of the torus two circuits cross, the vertical one being crossed always by the horizontal one, for instance, from left to right. A (polygonal) arc on the torus is monotone if it crosses horizontal circles from bottom to top. In particular, the vertical circuits are examples of monotone arcs. The definition of horizontal circles is easily extended to the case when the horizontal direction is not necessarily parallel to a side of $\mathrm{Q}$. Monotone arcs are defined accordingly. An upward drawing of a toroidal map $M$ is a map equivalent to $M$ drawn on the torus with monotone (polygonal) arcs and at each vertex $v$ of $M$ at least one edge incident to $v$ enters $v$ from below (with respect to the chosen horizontal direction) and at least one edge enters $v$ from above (see Figure 1(a)). Obviously, an upward drawing defines an upward orientation of $M$.

\section{A flow model for upward orientations}

An upward distribution of a toroidal map $M$ is a particular partition of its angles. It can be defined as a labelling by 0 's or 1's of the angles of $M$ such that there are exactly two 1's at each vertex and exactly two 0's at each face. It is obvious that an upward orientation of $M$ induces an upward distribution of $M$. However, the converse is not true. On the other hand, there is an easy algorithm to check whether a given upward distribution is induced by an upward orientation.

There are two papers in the graph drawing literature $[2,10]$ approaching geometrical representations by means of a flow model. The present model and the two preceding ones have only linear programming as a common feature.

An upward distribution consists of assigning $2 n$ units to the vertices of $M$ at the rate of exactly two per vertex, to route them from each vertex toward the adjacent faces through the angles at the rate of at most one per angle, and in such a way to recollect at each face $f$ of degree $d(f)$ exactly $d(f)-2$ units $[3,8,9]$.

Given a toroidal map, we define a network $K(M)=(X, U)$ as follows. The vertex set $X$ of $K(M)$ consists of vertices $V \cup F$ of the angle graph $\mathcal{A}$ of $M$ and two extra vertices, the source $S$ and the sink $T$. Edges among vertices in $V \cup F$ are the same as in the angle graph, and the source $S$ is connected to each vertex of $V$ (the vertices of $M$ ) and the sink $T$ is connected to each vertex of $F$ (the faces of $M$ ). The edges of $K(M)$ are oriented, from $S$ to $V$, from $F$ to $T$, and from $V$ to $F$. The network $K(M)$ contains an extra edge $\alpha$ with the initial vertex 
$T$ and the terminal vertex $S$. Each edge $e$ of the network $K(M)$ has associated an interval $\left[a_{e}, b_{e}\right]$ of real numbers. We will consider flows in $K(M)$, and $a_{e}$ and $b_{e}$ will represent the lower and the upper bound on the flow through the edge $e$. The intervals $\left[a_{e}, b_{e}\right]$ are defined as follows:

- If $e=\alpha$, then the interval is $[2|V|, \infty]$.

- If $e=(S, v), v \in V$, the interval is [2,2].

- If $e=(f, T), f \in F$, the interval is [ $d(f)-2, d(f)-2]$.

- If $e=(v, f), v \in V, f \in F$, the interval is $[0,1]$.

An integral flow $\varphi$ in $K(M)$ defined on $U$ is feasible if $\varphi(e) \in\left[a_{e}, b_{e}\right]$ for every edge $e \in U$. A direct consequence of the definitions is the following.

Proposition 1. Given a map $M$ and its capacitated network $K(M)$, there is a one to one mapping between the feasible flows on $K(M)$ and the upward distributions of $M$.

Let us notice that the max-flow min-cut algorithm can be used to obtain a feasible flow on $K(M)$ in polynomial time if such a flow exists. The advantage of this method is that it can be adjusted in such a way that it works also in the case when some angles have a prefixed value. Another nice feature is that while a solution has been obtained, that is an indegree-two orientation of $\mathcal{A}$, all the other solutions including the ones which generate an orientation of $M$, can be obtained by inverting repeatedly the orientation of the edges of $\mathcal{A}$ along a circuit. This fact is a graph consequence of a classical property of linear programming.

\section{A topological interpretation of the Hoffman's feasibility conditions}

A cocycle $\omega$ of a graph $K=(X, U)$ is a set of edges $E(Y, \bar{Y})$ for some complementary subsets $Y$ and $\bar{Y}$ of $X$. If the graph $K$ is oriented, then every cocycle $\omega=E(Y, \bar{Y})$ can be written as

$$
\omega=\omega^{+}+\omega^{-}
$$

where $\omega^{+}$are those edges from $\omega$ that are oriented from $Y$ to $\bar{Y}$, and $\omega^{-}$are the edges directed from $\bar{Y}$ to $Y$.

Hoffman proved[1] that, given a network $K=(X, U)$, capacitated by intervals $\left[a_{e}, b_{e}\right], e \in U$, there exists a feasible flow in $K$ if and only if for each (minimal) cocycle $\omega$ the following conditions are satisfied:

$$
\sum_{e \in \omega^{+}} a_{e} \leq \sum_{e \in \omega^{-}} b_{e}
$$

and

$$
\sum_{e \in \omega^{-}} a_{e} \leq \sum_{e \in \omega^{+}} b_{e} .
$$

We shall refer to conditions (2) and (3) as Hoffman's conditions. 
Theorem 2. A toroidal map $M$ which admits an upward orientation is essentially 2-connected.

Below we sketch a relation between Hoffman's conditions and the characteristic property (1) of essentially 2-connected maps that, in particular, implies Theorem 2 since an upward orientation of $M$ yields an upward distribution, and then this implies the existence of a flow solution in $K(M)$ according to Proposition 1. Hoffman's conditions are therefore satisfied, and appear as follows. If $\omega$ is a cocycle of $K(M)$ corresponding to the partition $(Y, \bar{Y})$ of $X$, then condition (3) can be expressed as condition (2) for the same cocycle but viewed as being associated with the partition $(\bar{Y}, Y)$. Therefore it suffices to consider the following two cases

(i) $S, T \notin Y$, and

(ii) $S \in Y, T \notin Y$.

Let $V_{1}=Y \cap V$ and $V_{2}=V \backslash V_{1}$. Similarly we partition $F$ into $F_{1}=Y \cap F$ and $F_{2}=F \backslash F_{1}$. Then we can write $(Y, \bar{Y})$ in case (i) as

$$
\begin{aligned}
& Y=V_{1} \cup F_{1} \\
& \bar{Y}=V_{2} \cup F_{2} \cup\{S, T\}
\end{aligned}
$$

and in case (ii) as

$$
\begin{aligned}
& Y=V_{1} \cup F_{1} \cup\{S\} \\
& \bar{Y}=V_{2} \cup F_{2} \cup\{T\}
\end{aligned}
$$

Euler's formula implies that for a toroidal angle map

$$
|E(V, F)|=2(|V|+|F|) .
$$

Clearly,

$$
\left|E\left(V, F_{1}\right)\right|=\sum_{f \in F_{1}} d(f) .
$$

Let us first consider case (i). By (4) and (5), (2) is equivalent to

$$
\left|E\left(V, F_{1}\right)\right|-2\left|F_{1}\right| \leq 2\left|V_{1}\right|+\left|E\left(V_{2}, F_{1}\right)\right|
$$

which simplifies to

$$
2\left(\left|V_{1}\right|+\left|F_{1}\right|\right)-\left|E\left(V_{1}, F_{1}\right)\right| \geq 0 .
$$

Similarly we have for (3)

$$
2\left|V_{1}\right| \leq\left|E\left(V_{1}, F_{2}\right)\right|+\left|E\left(V, F_{1}\right)\right|-2\left|F_{1}\right|
$$

or

$$
2\left(\left|V_{2}\right|+\left|F_{2}\right|\right)-\left|E\left(V_{2}, F_{2}\right)\right| \geq 0 .
$$

In case (ii), (2) is trivial due to the infinite upper capacity $b_{\alpha}$ of $\alpha$, and (3) can be expressed as:

$$
2|V| \leq 2\left|V_{2}\right|-2\left|F_{1}\right|+\left|E\left(V_{1}, F_{2}\right)\right|+\left|E\left(V, F_{1}\right)\right| .
$$




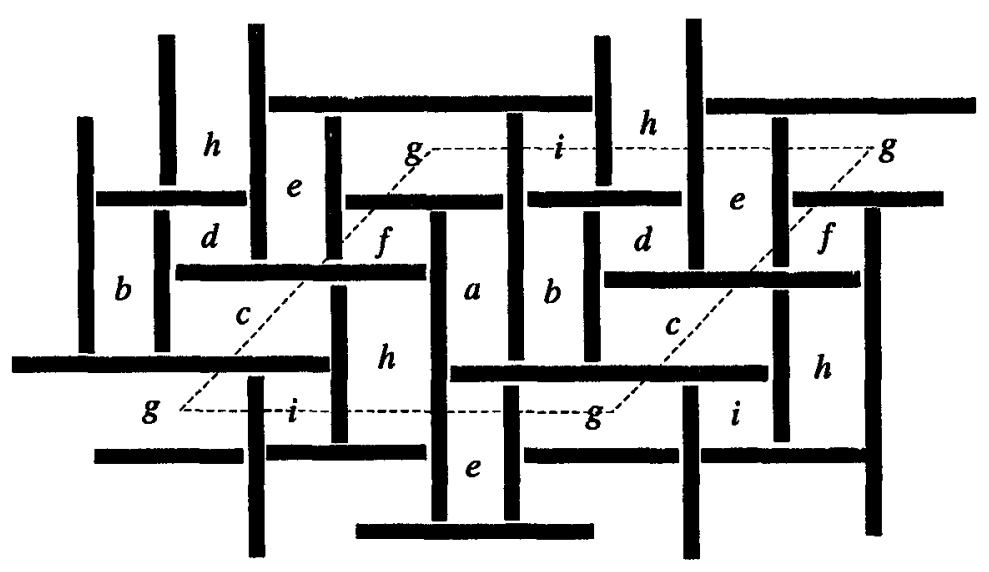

Fig. 2. A tessellation representation of $M$ and $M^{*}$

This is equivalent to

$$
2\left(\left|V_{2}\right|+\left|F_{2}\right|\right)-\left|E\left(V_{2}, F_{2}\right)\right| \geq 0 .
$$

In both cases, the Hoffman's conditions on $K(M)$ appear to be equivalent to the system of inequalities (1) defining the essential 2-connectivity of $M$. Conversely, the essential 2-connectivity of $M$ appears to be sufficient for constructing an upward drawing of $M$, therefore also an upward orientation of $M$. The effective construction of an upward drawing of an essentially 2-connected toroidal map relies on the construction of a tessellation representation, obtained by a reduction process of essentially 2-connected maps. Details on these results are presented in [6]. (Tessellation representations of plane graphs are studied in [11].) In Figure 2 we show the tessellation representation of the map $M$ from Figure 1(a). One has to consider only the part within the dotted fundamental polygon $\mathrm{Q}$ of the torus. The horizontal line segments represent the vertices of $M$, the vertical segments are the faces of $M$, and the quadrangles represent corresponding edges. All the incidences are preserved.

Theorem 3. A toroidal map $M$ admits an upward drawing if and only if $M$ is an essentially 2-connected map.

\section{References}

1. BERGE Claude, GHOUILA-HOURI A., Programmes, jeux et réseaux de transport, Dunod, 1962.

2. BERTOLAZZI P., Di BATTISTA G., On Upward Drawing Testing of Triconnected Digraphs, Proc. 7th ACM Symposium on Computational Geometry, 1991, 272-280. 
3. BOUSSET Marc, Orientation d'un schéma par passage d'un flot dans les angles, thèse de doctorat, E.H.E.S.S., 1993.

4. Di BATTISTA G., TAMASSIA R., Algorithm for plane representations of acyclic digraphs, Theoret. Comput. Science 61 (1988) 175-198.

5. GROSS J. L., TUCKER T. W., Topological Graph Theory, John Wiley and Sons, 1987.

6. MOHAR B., ROSENSTIEHL P., Tessellation and visibility representations of maps on the torus, preprint.

7. OSSONA de MENDEZ P., Orientations bipolaires, thèse de doctorat, E.H.E.S.S., 1994.

8. ROSENSTIEHL P., Flot sur les angles et orientations d'une carte, in Table ronde "Cartes et hypercartes", A. Machi Ed., LITP 92.80, 1992.

9. ROSENSTIEHL P., Embedding in the plane with orientation constraints: The angle graph, Annals New York Academy of Sciences, 1989.

10. TAMASSIA R., On embedding a graph in the grid with the minimum number of bends, SIAM J. Computing 16 (1987) 421-444.

11. TAMASSIA R., TOLLIS I. G., Tessellation representation of planar graphs, in Proc. Twenty-Seventh Annual Allerton Conference on Communication, Control and Computing (1989) 48-57. 